\title{
Estudo de caso: prevenção de recaída para criança e adolescente enuréticos com remissão espontânea
}

\author{
Rodrigo Fernando Pereira \\ Universidade de São Paulo \\ Edwiges Ferreira de Mattos Silvares \\ Instituto de Psicologia da Universidade de São Paulo
}

\begin{abstract}
RESUMO
A literatura aponta o tratamento comportamental com alarme como o mais eficaz para os casos de enurese noturna. Quando um procedimento comportamental adicional, como a superaprendizagem, é adicionado a este tratamento, as taxas de recaída são reduzidas. Neste procedimento há um gradual aumento de ingestão de água imediatamente antes de dormir. Possivelmente o maior controle obtido deve-se a essa combinação atuar tanto nos níveis plasmáticos de vasopressina como na capacidade funcional da bexiga. Os dois casos apresentados são de enuréticos que haviam adquirido o controle recentemente, mas suas famílias demandavam um atendimento para diminuir as chances de recaída. Utilizou-se, então, o alarme conjugado à superaprendizagem desde o início do atendimento, com a finalidade de se estabelecer um controle completo. No primeiro caso, de uma adolescente de dezoito anos, não houve molhadas durante o tratamento. No segundo, de um garoto de oito anos, as molhadas voltaram com a ingestão de líquido e tornaram a cessar, possivelmente por ter sido obtido um controle maior por parte da criança. Acredita-se que o procedimento descrito possa ser útil nos casos de crianças que deixaram recentemente de ser enuréticas ou não alcançam o critério para diagnóstico.
\end{abstract}

Palavras-chave: enurese noturna; prevenção de recaída; superaprendizagem.

\section{ABSTRACT \\ Case study: relapse prevention with recently continent enuretic child and adolescent}

Behavioral treatment for enuresis using urine alarm is the most efficient way to treat bedwetting, according to the literature. Using an additional behavioral procedure such as overlearning can reduce the relapse rate to $10 \%$. This procedure implies a gradual increase of water ingestion few moments before bedtime. Possibly the better control was obtained due to combined results of increased vasopressin levels and increased bladder functional capacity. The case studies described are enuretics who have recently achieved nocturnal bladder control, whose families demanded treatment for relapse prevention. Urine alarm and overlearning were used from the beginning of treatment. In the first case, of an eighteen year-old female, there were no more bedwetting episodes during the treatment. In the second case, of an eight year-old boy, relapsed when overlearning was inserted and reached dryness later even with fluid ingestion, suggesting a stronger control. We believe that this procedure can be useful for prevention in cases where children recently became dry or their bedwetting frequency does not fit enuresis criteria.

Keywords: nocturnal enuresis; relapse prevention; overlearning.

A enurese noturna é um dos quadros clínicos mais comuns na infância e na adolescência, afetando cerca de $10 \%$ das crianças de seis anos e $2 \%$ dos jovens adultos com 18 anos (Butler, 2004). A cada ano, cerca de $15 \%$ dos enuréticos param de molhar a cama sem nenhum tipo de tratamento (Fortsythe \& Redmond, 1989; Monda \& Hussmann, 1995). Esse fenômeno é chamado de remissão espontânea.
Estabelecer se uma criança é enurética ou não pode não ser tão simples, devido à diferença nos critérios. O que há de comum nos manuais de classificação é que deve haver molhadas em locais inadequados e essas molhadas não podem ser resultado de uma condição clínica geral. A questão é a freqüência na qual as molhadas ocorrem e a idade para diagnóstico. O DSM-IV (American Psychiatric Association, 2002) 
coloca como norma a existência de duas molhadas semanais em pelo menos três meses a partir dos cinco anos. Ainda assim, deixa aberta a possibilidade para o diagnóstico quando há freqüência menor, mas grande prejuízo para a criança. Já o CID-10 (Organização Mundial da Saúde, 1993) estabelece um critério confuso no qual fala em idade mental de quatro anos. Além disso, inúmeros estudos sobre enurese utilizam critérios próprios, aumentando as divergências (Butler, Robinson, Holland \& Doherty-Williams, 2004). Para este trabalho, adotamos o critério da APA.

A enurese é causada por uma conjunção de fatores. Existe o aspecto genético, uma vez que gêmeos monozigóticos têm maior probabilidade de compartilhar a enurese do que gêmeos dizigóticos (Ooki, 1999). Fisiologicamente, o que ocorre pode ser descrito nos termos dos três sistemas descritos por Butler (2004). O primeiro sistema é a diminuição da quantidade de vasopressina durante a noite nos enuréticos. Isso causa uma produção exagerada de urina, o que provocaria as molhadas. Contudo, há autores, como Houts (2003) argumentando que apenas uma minoria dos casos de enurese é causada pela baixa produção de vasopressina. $\mathrm{O}$ segundo sistema é a instabilidade da bexiga. Crianças enuréticas podem ter bexigas que se contraem antes de estar completamente cheias. $\mathrm{O}$ terceiro sistema é relacionado à incapacidade de acordar em resposta aos sinais da bexiga. Enuréticos tendem a ter mais dificuldade para acordar em resposta a estímulos relevantes do que não-enuréticos. A enurese pode ser causada por um ou mais desses fatores. Ainda segundo Butler (2004), existem fatores psicossociais que podem estar relacionados à falta de controle urinário, como ambientes pobres, falta de amamentação e pais intolerantes.

Os tratamentos mais comuns para enurese são os comportamentais e medicamentosos. O alarme é o tratamento psicológico mais efetivo, enquanto o DDAVP, ou desmopressina, um similar sintético do hormônio vasopressina, é o que obtém melhores resultados entre os tratamentos médicos. Porém, existe diferença entre os trabalhos sobre tratamentos medicamentosos e psicológicos em relação aos critérios. Enquanto os médicos medem a diminuição no número de molhadas, os psicólogos focam o cessar da enurese em seus estudos (Butler, Robinson, Holland \& DohertyWilliams, 2004).

O aparelho de alarme é um dispositivo composto por duas partes: um sensor colocado sobre o colchão, conectado a uma unidade sonora. Quando a micção se inicia, a unidade sonora é ativada, acordando a criança. Embora o tratamento medicamentoso com DDAVP possa ser tão efetivo quanto o alarme nos primeiros meses, ele tem taxas de recaída mais altas quando o tratamento é suspenso (Monda \& Hussmann, 1995). Além disso, quando um procedimento comportamental adicional é utilizado em conjunto com o tratamento com alarme, as taxas de recaída são ainda menores (Glazener, Evans \& Peto, 2005).

Um desses procedimentos adicionais é a superaprendizagem, que reduz a recaída de $40 \%$ para $10 \%$ (Houts, 2003). Após a obtenção de controle, a criança passa a beber diariamente uma determinada quantidade de água antes de deitar, quantidade essa que é estabelecida de acordo com sua idade, como mostra a Tabela 1. A quantidade é aumentada em cerca de 20 ou $30 \mathrm{ml}$ a cada duas noites consecutivas que a criança permanece seca. Após atingir o nível máximo de água, espera-se até que a criança obtenha 14 noites secas consecutivas e encerra-se o procedimento. Acredita-se que esse procedimento aumenta a capacidade funcional da bexiga, já que os valores são relacionados à capacidade funcional normal esperada para cada idade.

Tabela 1: Quantidades iniciais e finais de líquidos administradas na superaprendizagem (Butler, 2004)

\begin{tabular}{ccc}
\hline Idade (em anos) & Quantidade inicial (em ml) & Quantidade final (em ml) \\
\hline 6 & 113 & 227 \\
7 & 142 & 255 (1 copo americano) \\
8 & 170 & 284 \\
9 & 198 & 312 \\
10 & 227 & 340 \\
11 & $255(1$ copo americano $)$ & $369(1,5$ copo americano $)$ \\
12 & 284 & 397 \\
13 & 312 & 426 \\
14 & 340 & 454 \\
15 & $369(1,5$ copo americano) & 483 \\
16 & 397 & 511 \\
17 & 426 & 539 \\
18 & $454^{*}$ & $568^{*}$ \\
\hline
\end{tabular}

* Valores máximos para idades maiores de 18 anos 
A formulação de uma hipótese psicológica consensual sobre como o tratamento de alarme ou até mesmo a remissão espontânea ocorrem ainda está em aberto. A proposta mais aceita é da esquiva condicionada (Houts, 2003), na qual o alarme é um estímulo a ser evitado pela criança, através do acordar antes da micção, que é associado à bexiga cheia, ou contraindo a musculatura pélvica para evitar a molhada e dormindo a noite toda. A remissão espontânea poderia seguir o mesmo processo, no qual o estímulo a ser evitado é o incômodo de estar molhado. Porém, como isso tardou a acontecer, outros fatores podem estar envolvidos.

A partir da teoria dos três sistemas, pode-se especular que a remissão espontânea ocorre por uma normalização nos níveis de vasopressina durante a noite. Porém, outros aspectos podem permanecer problemáticos, como a baixa capacidade funcional, associada à instabilidade da bexiga. Daí o número alto de recaídas no tratamento e a possibilidade de crianças que já foram enuréticas voltarem a molhar frente a dificuldades emocionais, por exemplo (Butler, 2004).

Durante o nosso trabalho de pesquisa no Projeto Enurese, desenvolvido no Instituto de Psicologia da Universidade de São Paulo, tivemos a oportunidade de utilizar um procedimento diferente nos casos a serem apresentados posteriormente. Geralmente os casos atendidos são de crianças e adolescentes que se encaixam claramente no critério para enurese: com molhadas em pelo menos duas noites da semana, sem nunca terem estado secos. Utiliza-se o alarme e o acompanhamento psicológico, realizado semanalmente. Há os formatos individual e grupal, no qual também há um trabalho de orientação de pais realizado sistematicamente.

No Projeto Enurese, os critérios utilizados para avaliar o resultado dos tratamentos são (adaptado de Butler, 1994; 2004):

Sucesso inicial: 14 noites consecutivas sem molhadas.

Sucesso continuado: sem recaída por um período de seis meses após o sucesso inicial.

Sem sucesso: falha em obter o sucesso inicial, excluindo-se as desistências.

Recaída: mais de duas noites com molhadas em duas semanas.

Desistências: faltas em dois encontros consecutivos ou abandono do tratamento.

Saída inevitável: abandono do tratamento por motivos de força maior (e.g. mudança de cidade, morte de parente próximo).
Todas as crianças e adolescentes atendidos passam por entrevista de triagem, na qual a severidade da enurese, entre outros fatores, é avaliada utilizando o instrumento de Blackwell (1989). Além disso, avaliase o grau de impacto da criança ou adolescente, através da entrevista semi-estruturada de Butler (1994) e a intolerância dos pais, medida pela escala desenvolvida por Morgan e Young (1975). Depois disso, elas passam por um período de espera na qual aguardam atendimento. Nos dois casos objeto de estudo do presente trabalho, a enurese diminuiu consideravelmente ou até cessou no período entre a triagem e o atendimento. Ofereceu-se às famílias um atendimento a fim de verificar se o controle obtido era estável e reduzir a possibilidade de recaída. Utilizou-se o alarme, mesmo não havendo molhadas, combinado com a superaprendizagem. A intenção era aumentar a capacidade funcional da bexiga para garantir que ela suportaria grandes quantidades de líquido; caso houvesse molhadas nesse procedimento, o alarme serviria para a discriminação dos sinais da bexiga e conseqüente condicionamento.

Durante o atendimento, as famílias concordam em seguir as regras do atendimento no Projeto Enurese:

- Comparecer às sessões de acompanhamento do tratamento semanalmente.

- Preencher diariamente um registro de molhadas fornecido pelo terapeuta e entregue na sessão semanal.

- Utilizar o alarme todas as noites e testá-lo semanalmente para verificar seu funcionamento.

- Controlar a ingestão de líquidos diuréticos duas horas antes de dormir, como chá, refrigerante, café, leite achocolatado.

- Ingerir as quantidades de água determinadas no período da superaprendizagem.

Essas regras são as mesmas para atendimentos grupais ou individuais. Nos atendimentos individuais, o terapeuta atende a criança, os pais ou a família toda para acompanhamento do tratamento de acordo com as necessidades de cada caso. Já nos atendimentos grupais, as sessões são divididas sistematicamente num momento exclusivo com as crianças e outro de orientação aos pais.

\section{CASO 1: B. C.}

B. era uma adolescente que havia acabado de completar 18 anos. Sempre havia sido enurética, até o momento da avaliação. Antes do atendimento, ela 
havia viajado com a escola para comemorar a formatura no segundo grau e no seu retorno seus pais haviam reformado seu quarto. Segundo a adolescente, não houve molhadas durante a viagem. $\mathrm{O}$ controle persis- tiu por algumas semanas com o quarto novo, momento no qual ela foi chamada para atendimento. Algumas molhadas ocorreram, mas ainda não o suficiente para classificá-la como enurética.

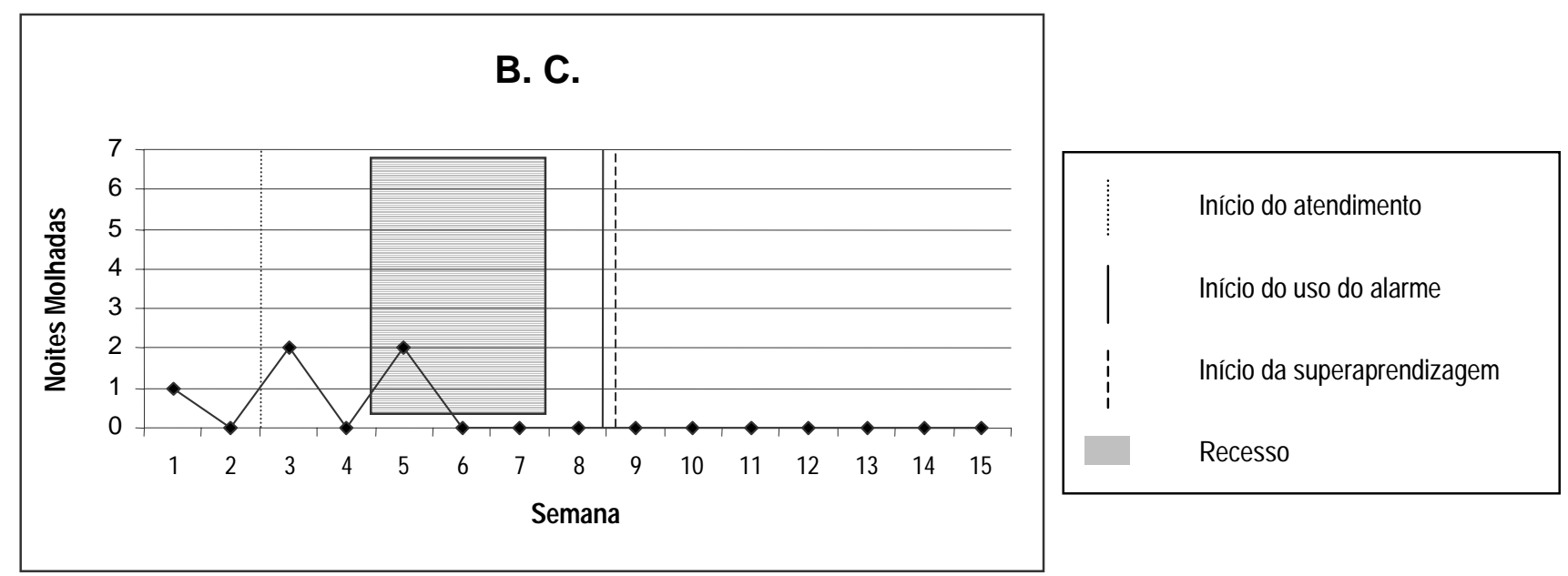

Figura 1: Freqüência de molhadas da adolescente B. C.

Como pode ser observado na Figura 1, antes do atendimento, a adolescente teve apenas uma molhada em duas semanas. Logo no início das sessões, houve quatro molhadas em três semanas. Houve um recesso do atendimento; no retorno, a adolescente tinha completado três semanas sem molhadas, mesmo antes de iniciar o uso do alarme. Optou-se por introduzir o alarme conjugado com a superaprendizagem, como descrito acima. A adolescente iniciou o trabalho com $450 \mathrm{ml}$ de água, aumentando gradativamente até $570 \mathrm{ml}$, correspondente à capacidade funcional normal para sua idade. Essa quantidade foi atingida na décima segunda semana, a partir da qual se contou catorze dias em que não houve molhadas. Após esse período, a adolescente continuou o uso do alarme sem nenhum tipo de controle dos líquidos, e sem a ingestão de água obrigatória específica da superaprendizagem. Esse é um período de teste em que a criança ou adolescente pode verificar sua capacidade e, como frequentemente é bem sucedido, permanecer ainda mais confiante da aquisição do controle. Finalizado esse momento, a adolescente recebeu alta e foi desligada do programa.

A análise exclusiva desse caso não permite verificar se o procedimento é eficaz na prevenção de recaída. A adolescente não teve mais molhadas, mas não se sabe se elas ocorreriam sem o atendimento. A hipótese é de que não ocorreriam, pois o controle adquirido por ela parece ter sido bastante forte, suportando até mesmo as grandes quantidades de água ingeridas na superaprendizagem. Contudo, ainda existe a possibilidade da superaprendizagem ter auxiliado no fortalecimento do controle: ao dormir com a bexiga cheia além do normal, a adolescente pode ter tido que lidar com os sinais recebidos do órgão durante a noite, reagindo com contração da musculatura pélvica e relaxamento do músculo detrussor, uma vez que ela conseguia ficar a noite inteira seca sem acordar para urinar.

\section{CASO 2: F. R.}

F. era um garoto de oito anos de idade que obteve o controle total no período que precedeu o tratamento. Ainda assim, persistia por parte dos pais uma preocupação em relação à estabilidade desse controle, motivo pelo qual eles solicitavam atendimento. Inserimos F. num grupo de atendimento a crianças da mesma faixa etária que já estava em andamento há algumas semanas. A adaptação de F. foi boa, sendo bem aceito pelo grupo e participando imediatamente das atividades. Da mesma forma, a participação dos pais na sessão de orientação não foi prejudicada pelo ingresso tardio.

Após algumas semanas participando do atendimento e ainda sem molhadas, o alarme foi entregue para a criança, juntamente com as instruções para a superaprendizagem, que se iniciou com uma quantidade de $170 \mathrm{ml}$. 


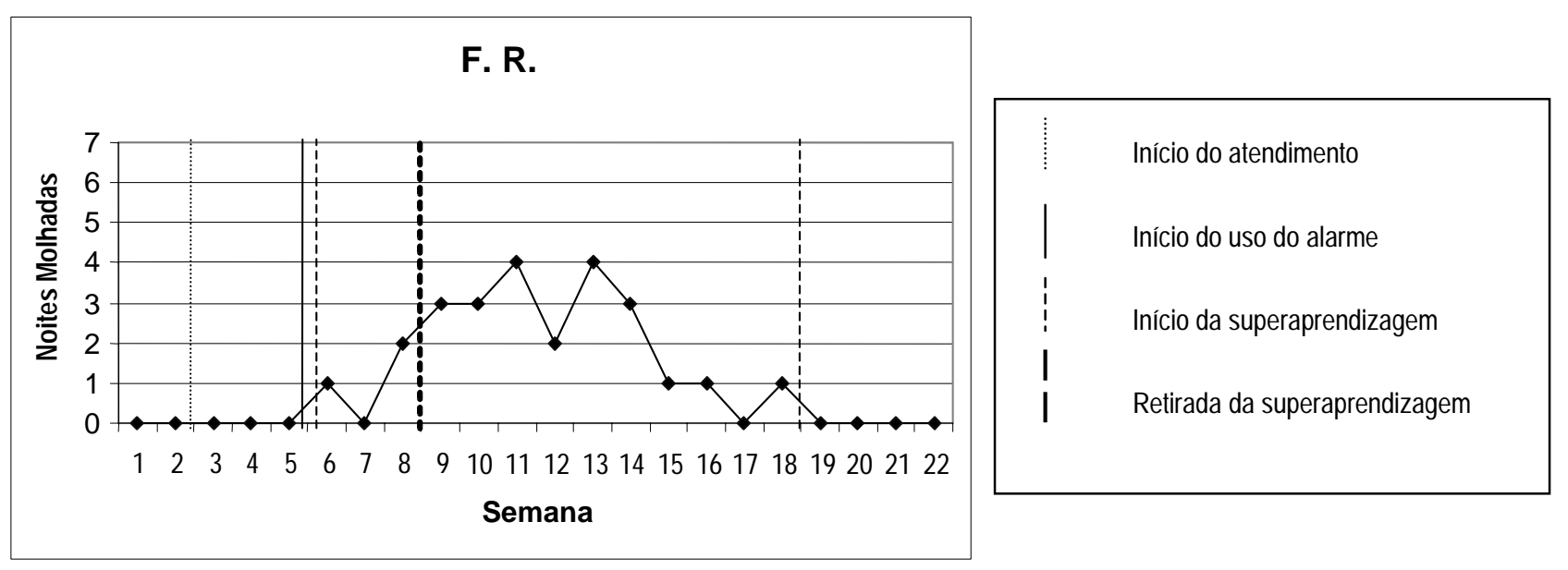

Figura 2: Freqüência de molhadas de F. R.

A Figura 2 mostra o desenvolvimento das molhadas da criança. Nas duas primeiras semanas, a quantidade de noites em que houve o xixi na cama ficou dentro do esperado, e a criança conseguiu um aumento gradual na quantidade de água. Porém, a partir da quantidade atingida na terceira semana, o número de molhadas aumentou consideravelmente, levando o terapeuta a suspender a superaprendizagem. Além da superaprendizagem, outros fatores podem ter influenciado no aumento das molhadas, como a temperatura, que caiu durante o período e a mudança de rotina, uma vez que se passava por férias escolares. As molhadas persistiram por diversas semanas mesmo sem a ingestão de água antes de deitar, o que preocupou a família. Nesse período a criança utilizou o alarme normalmente.

Após algumas semanas, as molhadas voltaram a diminuir e a criança obteve 14 noites secas consecutivas, critério para sucesso inicial e reinserção da superaprendizagem. Houts (2003) coloca que o procedimento pode ser tentado por oito semanas, após as quais, caso a criança não obtenha o controle com a ingestão de água, deve-se voltar a esperar que ela fique duas semanas seca sem a superaprendizagem e desliga-se a família do tratamento. A criança começou novamente a ingerir água a partir da quantidade mínima $(170 \mathrm{ml})$. Dessa vez, porém, ela não voltou a molhar, chegando à quantidade máxima e finalmente obtendo 14 noites secas consecutivas com essa quantidade, recebendo alta.

O que se pode hipotetizar nesse caso é que o controle adquirido por F. R. espontaneamente foi um controle fraco, que não lhe deu condições de lidar com quantidades maiores de líquido à noite. A criança, antes do atendimento, pode ter tido um aumento nos níveis plasmáticos de vasopressina, diminuindo a produção de urina à noite. Contudo, a baixa capacidade funcional e, conseqüentemente, a instabilidade da bexiga podem ter persistido, motivo pelo qual uma maior quantidade de urina provocava os episódios enuréticos. Acredita-se, ainda, que o uso do alarme e a segunda tentativa de superaprendizagem tiveram como resultado o aumento dessa capacidade, fortalecendo o controle e encerrando definitivamente as molhadas.

\section{CONSIDERAÇÕES FINAIS}

O tratamento com alarme para enurese noturna é amplamente difundido na literatura e apontado como o mais efetivo a longo prazo, principalmente quando associado à superaprendizagem, que reduz as taxas de recaída para $10 \%$ (Houts, 2003), possivelmente por aumentar a capacidade funcional da bexiga e controlando a instabilidade detrussora (Butler, 2004). Recebemos no nosso projeto dois casos que não se encaixavam nos critérios para enurese, mas pela demanda da família e para conter possíveis prejuízos de uma enurese mal controlada, utilizamos o alarme mesmo sem haver molhadas, já com o procedimento da superaprendizagem. Observando conjuntamente os dois casos, pode-se verificar que o procedimento pôde ajudar a confirmar que o controle obtido é estável, dando tranqüilidade e confiança para a família e a criança de que dificilmente o problema voltará a acontecer. Além disso, nos casos em que esse controle não foi adquirido completamente, o procedimento 
sugerido fará surgir essa deficiência - que, a nosso ver, ocorreria mais cedo ou mais tarde - e dá a chance de resolvê-la. Argumenta-se, então, que esse procedimento pode ser uma fonte de prevenção de futuros problemas para crianças enuréticas e que deixaram de sê-lo recentemente ou que não alcançam os critérios de frequiência suficientes para o diagnóstico.

Contudo, para se verificar melhor a eficácia desse procedimento, pesquisas mais aprofundadas poderiam ser realizadas. O procedimento poderia ser aplicado em uma amostra maior, com um acompanhamento mais longo e com um grupo controle formado por crianças recém-continentes que não passaram pelo tratamento. Por outro lado, poderia se fazer estudos de casos que verificassem mais a fundo as condições da capacidade funcional da bexiga e da quantidade de vasopressina secretada durante a noite antes e depois do tratamento, o que foi uma limitação deste trabalho.

\section{REFERÊNCIAS}

American Psychiatric Association (2002). Manual diagnóstico e estatístico de transtornos mentais. Porto Alegre: Armed.

Blackwell, C. (1989). A guide to enuresis: A guide to a treatment of enuresis for professionals. Bristol: Enuresis Resource and Information Center.

Butler, R. J. (1994). Nocturnal enuresis: The child's experience. Oxford: Butterworth-Heinemann.
Butler, R. J. (2004). Childhood nocturnal enuresis: Developing a conceptual framework. Clinical Psychology Review, 24, 909931

Butler, R. J., Robinson, J. C., Holland, P. \& Doherty-Williams, D. (2004). An exploration of outcome criteria in nocturnal enuresis treatment: The way forward. Scandinavian Journal of Urology and Nephrology, 38, 196-206.

Glazener, C. M. A., Evans, J. H. C. \& Peto, R. E. (2005). Alarm interventions for nocturnal enuresis in children. The Cochrane Database of Systematic Reviews, 2, Art. no.: CD002911.pub2.

Houts, A. C. (2003). Behavioral treatment for enuresis. Em A. E. Kazdin \& J. R. Weisz, (Orgs.), Evidence-based psychotherapies for children and adolescents (pp. 389-406). New York: Guilford Press.

Monda, J. M. \& Husmann, D. A. (1995). Primary nocturnal enuresis: A comparison among observation, imipramine, desmopressin acetate and bed-wetting alarm systems. The Journal of Urology, 154(2), 745-748.

Morgan, R. T. T. \& Young, G. C. (1975). Parental attitudes and the conditioning treatment of childhood enuresis. Behavior Research and Therapy, 13, 197-199.

Ooki, S. (1999). The genetic-epidemiologic analysis of nocturnal enuresis in childhood: Estimation of genetic and environmental factors by means of covariance structure analysis. Japanese Journal of Health and Human Ecology, 65, 297-310.

Organização Mundial de Saúde. (1993). Classificação de transtornos mentais e de comportamento da CID-10. Porto Alegre: Artmed.

\section{Sobre os autores:}

Rodrigo Fernando Pereira: Piscólogo (Universidade Mackenzie). Mestre em Psicologia Clínica (Universidade de São Paulo).

Edwiges Ferreira de Mattos Silvares: Professora Titular do Departamento de Psicologia Clínica do Instituto de Psicologia da Universidade de São Paulo.

Endereço para correspondência: Edwiges Ferreira de Mattos Silvares - Av. Prof. Mello Moraes, 1721 - Bloco F - Sala 30 - 05508-030 Cidade Universitária - São Paulo - Endereço eletrônico: efdmsilv@ usp.br. 